\title{
A STUDY OF COSTS AND BEHAVIORAL OUTCOMES OF MENSTRUAL REGULATION SERVICES IN BANGLADESH
}

\author{
BONNIE J. KAY ${ }^{1}$ and SANDRA M. KaBIR 2 \\ ${ }^{1}$ Department of Public Health Policy and Administration, School of Public Health. University of \\ Michigan, 1420 Washington Heights, Ann Arbor, MI 48109-2029, U.S.A. and 'Bangladesh Women's
} Health Coalition, Dhaka, Bangladesh

\begin{abstract}
This paper reports the results of a program evaluation of menstrual regulation (MR) services provided by the Bangladesh Women's Health Coalition, a nongovernmental organization formed in response to a concern about the availability of quality MR services to Bangladeshi women. The program emphasizes individual counseling which stresses informed choice in reproductive health care. The evaluation examines the cost of this process as a function of behavioral outcomes which include the percentage of clients who are post-MR contraceptive acceptors and the percentage which return for follow-up care and consultation 2 weeks after the procedure. The average cost per post MR contracepting client is $\$ 3.75$; the average cost per returning client is $\$ 5.68$, figures which appear to be well within the range of costs reported by family planning programs in developing countries.
\end{abstract}

Key words - menstrual regulation, cost-effectiveness, evaluation, Bangladesh, family planning

\section{INTRODUCTION}

Septic abortion continues to exact an enormous toll in terms of maternal mortality and morbidity in many developing countries. Estimates for Bangladesh place complications from septic abortion as the cause of at least $25 \%$ of maternal mortality or approx. 8000 deaths annually $[1-4]$. In addition, large numbers of women suffer severe morbidity and permanent sterility.

In an attempt to address these problems the government of Bangladesh encouraged the introduction of menstrual regulation (MR) services beginning in 1974 as one approach to dealing with unwanted pregnancies and as a back-up for contraceptive method failure. Despite a restrictive penal code in which induced abortion is considered legal only to save the mother's life, MR is not regulated by the code and is considered to be an 'interim method for establishing nonpregnancy' among women considered at risk of pregnancy $[1$, p. 2]*. By $1979 \mathrm{MR}$ was an official part of the national family planning program with the government committed to training physicians and paramedics in the procedure and delivering services through public hospitals and health and family planning facilitiest.

The demand for MR in Bangladesh has continued to increase since 1974 with present conservative annual estimates totaling close to 70,000 procedures [5]. This increase has occurred in the context of a politi-

*Menstrual regulation is aspiration evacuation of the uterus. It is usually performed manually in Bangladesh using a syringe and a hollow flexible plastic tube called a cannula. MR can be performed as early as a missed menstrual period can be established.

†Precise figures on how extensively MR is used in the developing world are not available, however, it is the method of choice in India where abortion is legal. India also manufactures and exports MR equipment to other areas in southeast Asia. cally turbulent international environment surrounding abortion and the funding of family planning programs. Political controversy often results in a focused concern by medical authorities with structural and medical aspects of care: sanitary facilities, adequate clinical performance and client safety [6]. In the developing world in particular, these concerns are primary and usually very difficult to resolve satisfactorily. Assuring that government is expending resources for medically safe MR procedures removes some controversy from this area and supports the continued availability of resources.

In Bangladesh considerable effort has been expended to monitor MR training programs and assess medical outcomes [5, 7]. The Bangladesh Association for the Prevention of Septic Abortion, the major organization conducting research on MR, has recently completed a national study for the purpose of determining the prevalence of formally trained MR providers, the availability of appropriate equipment, the extent of contraceptive counseling and the proportion and nature of medical complications. A geographically stratified sample of 2454 MR providers was interviewed. Two thirds of this sample had been formally trained in government training programs. Recommendations from this study received an enthusiastic response from government health officials. The report recommended extending training programs to female medical assistants and nurses.

While a focus on medical aspects of MR services is important it frequently becomes the sole concern of program evaluation efforts. Usually neglected are aspects of services which address long term reduction in the need for MR, such as the availability and effectiveness of client education and counseling about pre-conception family planning methods. Third World women collectively and individually have spoken to the importance of staff attitudes and demeanor toward clients and the degree to which advice and information is accepted and acted upon 


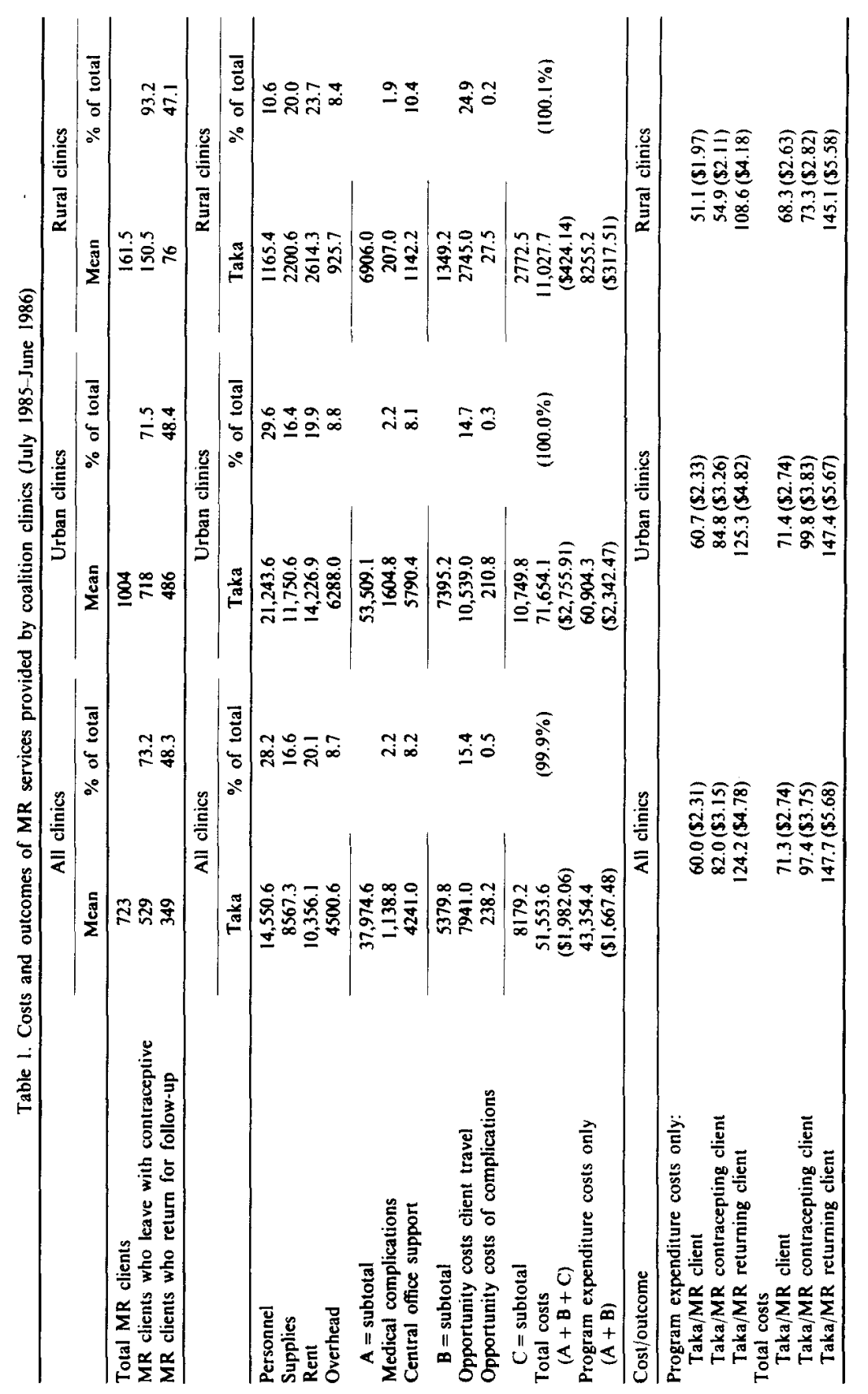


$[8,9]$. Nevertheless, these aspects of care are often dismissed as luxuries which are costly and beyond the scope of practical program implementation. They are also more difficult than structural and medical aspects to evaluate both theoretically and practically.

\section{THE BANGLADESH WOMEN'S HEALTH COALITION}

In 1980 the Bangladesh Women's Health Coalition (BWHC) was formed* in response to a concern about the availability of quality MR services to Bangladeshi women. As interpreted by BWHC, quality of care goes beyond assuring a minimally acceptable medical standard for cleanliness and clinical performance. Quality services also encompass the nature of the interaction between staff and clients: showing concern and respect for clients as individuals as well as structuring services to reflect this concern. Counseling is a key aspect of a client's visit. Along with providing encouragement to practice family planning its intent is to assist clients in making informed choices about their health. A related long-range aim is to raise the level of expectation of women, particularly poor women, about reproductive health services.

BWHC, a privately funded nongovernmental organization (NGO), is presently one of only 3 such sources of MR services in Bangladesh. It operates 6 clinics in both rural and urban communities and is considered to provide high quality reproductive health services. Although the coalition is small relative to government MR service delivery, it is important as a demonstration that a women-controlled organization can effectively organize and deliver quality reproductive health care services in a Third World setting where women have traditionally not enjoyed equal status, opportunities and responsibilities. By providing such services it seeks to legitimize quality of care as a policy issue for government consideration. A related issue, which this analysis addresses, is whether an investment in quality of care is costly and, if so, whether it is worth the expenditure of additional resources.

BWHC first developed and operated 3 clinics in urban areas in or near Dhaka (Dhaka, Narayanganj and Tangail). In 1983-84, with funds from the International Women's Health Coalition and a 3-year grant from the Ford Foundation, the remaining 3 clinics were established, Mirpur in suburban Dhaka, and Palash and Bolora which are in predominantly rural communities. Although the coalition initially established the clinics primarily as sources of MR services, the linkage between $M R$ and the subsequent practice of family planning has always been viewed as important and consistent with the long-range goal of reducing the need for MR services. The principle is one of reproductive choice and assuring that alternatives are available for women. The provision of a range of contraceptive methods (pill, IUD, foam/ condom, injectibles and referrals for sterilization)

*BWHC is affiliated with the International Women's Health Coalition, a New York-based organization which promotes the development of high quality reproductive health care programs for women in developing countries. soon became an integral part of services. Post MR contraceptive choices vary from clinic to clinic and refiect difference in preferences among clients. At Tangail, over $56 \%$ choose the IUD while at Narayanganj, less than $17 \%$ select this method, a majority choosing the pill. Condoms and injectibles are used by only $6.5 \%$ of clients at Tangail while the fraction is over $27 \%$ at the Narayanganj clinic.

Over the past 6 years, the scope of services has broadened to include prenatal care, maternal and child health care, general health including immunizations, and other gynecological care making the BWHC clinics a source of integrated reproductive and child health care services. By the time the newest clinics were operational, all were providing a full range of services. By 1986, $26.2 \%$ of client visits were for family planning (including MR), $30.6 \%$ were for general health and prenatal care while $43.2 \%$ of visits were for children. In general, MR clients comprise a relatively small fraction of total clients visiting the clinics with percentages ranging from $0.8 \%$ (Bolora) to $12.1 \%$ (Mirpur). While the 2 rural clinics have the lowest number of MR clients their overall caseloads are comparable to those of the urban clinics. Caseloads range from 8200 (Palash) to 15,400 (Dhaka). Generally the number and percentage of MR clients are stable (at the more established clinics) or increasing as in the case of Bolora. With an annual caseload of over 11,000 and the highest travel costs in terms of distance (walking 6 miles is not uncommon) many women come first to Bolora with children for immunizations and other child health needs. It appears likely that these visits serve to introduce women to the reproductive health services also available and help reduce the shyness prevalent in rural, often conservative communities concerning MR and family planning. The Dhaka clinic, the oldest and located in the most densely populated section of the city, has experienced a consistently increasing total caseload over its operation period but for the years 1984, 1985 and 1986, has provided annually decreasing number of MR procedures. This apparentiy was a result of a personnel problem at the clinic which has since been resolved. Latest figures suggest 1987 totals will again reflect the steadily increasing demand for MR experienced by all 6 clinics.

The allocation of resources devoted specifically to counseling makes the coalition's MR services organizationally different from government providers. All 6 clinics share a common process for interacting with clients and designate a specific staff member whose function it is to counsel. In addition, all staff have appropriate information and may respond to questions. During a clinic visit, several opportunities are provided for clients to get information, ask questions and discuss their choices with the counselor and with the paramedic who performs the MR. Approximately $20-25 \%$ of a client's contact time with clinic staff is devoted to counseling and information sharing.

This paper reports the results of a program evaluation which sought to examine the effects and costs of providing high quality MR services in a disadvantage environment. Specifically the evaluation assesses the costs and selected outcomes of a process of care which emphasizes individualized counseling concern- 
ing family planning and reproductive health, provides real choices among contraceptive methods, and promotes respect for the client. Direct program expenditures are of most interest to those involved in short-term operational program planning and we present these data as a function of program outcomes. However, while the focus of the analysis is a specific program, the perspective is one which examines the program as part of a broader social context. Thus we consider costs to be more than specific program expenditures and include the opportunity costs of the program's clients. The process through which services are provided, including the mode of service delivery, has associated costs to participants which may be reflected by different clinic utilization and contraceptive compliance rates. We consider clients who return to the clinic periodically for routine care, immunizations and family planning as investing in future health benefits through a reduction in unwanted births and the need for MR services. While counseling may add to costs by encouraging greater utilization in the short term, the use of well-health services presumably has a long-term benefit in the future reduction of illness associated with reproduction. The evaluation asked: How costly is quality reproductive health care in relation to outcomes which are consistent with reducing the long term need for MR services?

\section{PROGRAM EFFECTIVENESS}

Measures of program effectiveness relate to the general objective of reducing the long term need for MR services. Two measures were defined: (1) the percentage of MR clients who leave the clinic with a contraceptive method, and (2) the percentage of MR clients who return for a follow-up visit 2 weeks after the procedure as recommended during the counseling session. While both measures relate to short term behavior concerning reducing the necd for MR, they are necessary first steps in a long term process. In this sense, the follow-up visit can be considered a proxy for an active, contracepting client who is using the clinic as a source of reproductive health care services.

The validity of the follow-up visit as an effectiveness measure depends on its correlation with client attitudes and behaviors which promote initiating and continuing family planning and gynecological care. Counseling and generally supportive attitudes held by clinic staff are presumed to be connected with such behaviors $[10,11]$. An opportunity to informally test this presumption arose with the establishment of the first of the 3 newer clinics at Mirpur.

* $T$-test comparing the percentage of MR clients who returned for a follow-up visit for the 2 time periods. January-December, 1984 and July 1985-June 1986: $T$-value $=-5.54$, statistically significant at $P<0.0005$.

†In 4 clinics (Dhaka, Narayanganj, Tangail and Mirpur), administrators included estimates for staff physicians. In these clinics it is the practice to have MR clients briefly check with the physician before they leave the clinics. Times were on the order of $5 \mathrm{~min} /$ client. Because of the relatively high salaries of physicians, use of this resource contributes to the higher costs calculated for the urban clinics (see Table 1).
Staffing at the newer clinics was planned to conform with that of government MR facilities which did not include a specific staff member designated as 'counselor'. Initially counseling duties were shared among the paramedic staff. When it became apparent that insufficient time was being given to counseling by the paramedics due to the press of other clinical responsibilities. the clinic administrator assumed specific responsibility for counseling each client. This pattern is presently followed by all 3 of the newer clinics. Mirpur clinic more than doubled the percentage of clients returning for follow-up visits when counseling was organized and responsibility assumed by a specific member (an increase from $20.6 \%$ to $41.4 \%)^{*}$. While the follow-up visit could also be motivated by negative clinical side effects from the $M R$, the numbers of clients seen with complications are on the order of 1 or 2 of approx. 750 each month. Thus it seems reasonable to use the follow-up visit rate as a proxy for positive program outcomes. A recent reorganization of the client record system has made it possible to track the pattern and purpose of visits of individual clients longitudinally. Preliminary data suggest that MR clients do return to coalition clinics in significant numbers to use family planning and other services for themselves and their children.

\section{PROGRAM COSTS}

The cost analysis component of this study encompasses all resources used which are associated with a clinic visit for an MR procedure. This includes the value of personnel, clinical and contraceptive supplies and administrative overhead. The coalition maintains a central office which provides additional administrative and logistics support for all 6 clinics. Estimates of the fraction of these costs attributable to MR clients have been added. We have also included estimates of medical resources used in treating side effects of the procedure and the opportunity costs resulting from the time spent by clients at the clinic and in travel.

Personnel costs were calculated using estimates of each staff member's time spent with MR clients. For purpose of this study clinic administrators were requested to estimate (1) the total time an MR client spent in the clinic on a normal day and on a busy day, and (2) individual staff time spent with an MR client during the course of her visit. Involved staff were the receptionist, counselor, paramedic and ayah (aidehelper)t. A formal personnel time study was not conducted due to logistical constraints. However, the small scale of clinics as well as a management philosophy which promotes interaction between staff lend support to using the administrators' figures as valid estimates of personnel time spent with MR clients. All 6 clinics share a similar process for dealing with MR clients. Total time spent at the clinic ranged from $30 \mathrm{~min}$ to $1.5 \mathrm{hr}$ on a normal day and 1.5 to $3 \mathrm{hr}$ on a busy day. Time estimates for the counselor ranged from 10 to $20 \mathrm{~min}$ per client. Client loads vary across clinics so some variation in time is to be anticipated. Although the administrators made their estimates independently the values were reasonably similar. The sum of individual staff times were generally within the total clinic time estimates for normal and 
busy clinic days suggesting the estimates, overall, were reasonable and internally consistent. A sensitivity analysis was conducted using a range of time estimates for key personnel (the counselor and the paramedic) to assess the impact of uncertainty on total personnel costs and, subscquently, on total costs. Total personnel costs were calculated as follows:

$$
\left.\left.\sum_{i} \text { (minutes per client }\right)_{i j} \text { (wage rate) }\right)_{i j}
$$

$=$ total personnel cost $/ \mathrm{MR}$ client, .

For all $i=$ staff with direct MR client contact at clinic $j$.

Wage rate $=\mathrm{taka} / \mathrm{min}$.

Wages were taken from annual salary expenditure records for each clinic, assuming an average 20 working days per month, 12 months per year.

Supply costs included expenditures for cotton, gauze, alcohol, etc. and contraceptives. The analysis allocated $80 \%$ of clinical supply expenditures to MR clients under the assumption that MR was the only surgical service provided and required the heaviest use of disposable supplies. The value of contraceptives dispensed to MR clients was estimated as an average of costs for a 3 month supply of pills and condoms, a single injection of Depo-Provera and an IUD [12]. The coalition receives contraceptive supplies free of charge from the government and as such are not part of the coalition's budget. They are, nevertheless, resources consumed and thus are considered as part of total costs.

Administrative overhead costs for each clinic included salaries for the administrator, 2 guards and a sweeper/cleaner, office supplies, utilities, postage, laundry and rent. With the exception of 1 vacuum aspirator machine which was not in operation during the period of this analysis, all equipment for MRs is disposable and listed under clinical supplies. Clinic space used for MR clients was estimated at $25 \%$ for each clinic and this fraction of rent was attributed to MR expenditures. All other administrative costs were summed and multiplied by the fraction of total clients who were MR clients.

The costs of support and logistics resources from the coalition's central office were determined by estimating the fraction of staff time devoted to direct clinic support. These included personnel (the Executive and Medical Directors, the Deputy Directors for administration, finance and research, support staff), travel, supplies, utilities and rent. This sum was multiplied by each clinic's fraction of total clients who were MR clients and averaged for all clinics. Averages for urban and rural clinic subgroups were also tabulated.

While approx. $90 \%$ of MR clients seen at the coalition clinics are nonwage earners and work within the home [13], there are costs associated with time spent traveling to a clinic to receive medical care. Part of these costs are cultural; that is, a woman will take care of the health needs of all members of her family before herself. Personal medical care is sometimes looked upon as self-indulgent. Childcare and other household responsibilities must be shifted to other household members or children must accompany the client to the clinic. Most travel to coalition clinics by foot or by ricksha. Travel distances in the rural areas can be as large as 12 miles round trip. Opportunity costs associated with the time spent away from home were thus approximated using a wage rate for a worker whose duties encompassed household activities. An average of wages paid to clinic workers responsible for cleaning and assisting the nurse aide in patient care was used as a proxy wage. Urban clients were estimated to spend 1-half day away from normal activities. The figure used for rural clients was a full day. Total client time costs were calculated for each clinic by multiplying the corresponding wage rate by the total number of $\mathrm{MR}$ clients served during the analysis period.

Cost estimates for treating side effects are difficult to assess due to the lack of a comprehensive means to follow up MR clients. The predominant side effect is retained tissue which requires a return trip to the clinic and a repeated $M R$ procedure [7]. In a systematic national sample of MR providers and clients, self reports put the complication rate in the range of $1.7-15.0 \%$ of cases treated by paramedic providers [7]. Cases seen in the coalition clinics are cstimated to be in the lower end of this range. A rate of $3 \%$ was used to calculate the number of repeated procedures required and which resulted from retained tissue. This number was multiplied by the unit cost per MR for each clinic to obtain the total cost for treating side effects. The opportunity costs associated with travel for repeated procedures were calculated as described above and added to client travel costs. The sum of personnel, supplies, administrative overhead, central office support, treatment of medical complications and client time costs comprise total costs for MR services.

All expenditure data were abstracted from coalition financial records for the period July 1985-June 1986. MR utilization data, including MR clients who were contraceptive acceptors and those who returned for follow-up visits, were obtained from monthly reports filed by each clinic with the coalition central office for the same time period. Costs were calculated for each clinic as a function of (1) total MR clients treated, (2) MR clients who were contraceptive acceptors, and (3) MR clients who returned in 2 weeks for a follow-up visit. Comparisons were made between the 4 urban and 2 rural coalition clinics.

\section{FINDINGS}

Numerical results of the analysis are presented in Table 1. Numbers of clients served by the 6 clinics ranged from 109 to 1457 with an average of 723 per clinic during the 12 month analysis period.

\section{Effectiveness}

Over $73 \%$ of MR clients leave the clinics with a contraceptive method (pill, IUD, condom or injectible). This is conservative estimate. Clients who leave the clinic stating they intend to obtain contraceptives from other sources were not counted in this fraction. If included, the percentage is closer to $88 \%$. A small number (less than $4 \%$ of all users) are referrals from facilities which provide tubal ligations and return to these clinics for the sterilization procedure after their MR. The highest contraceptive acceptance rate 
$(93 \%)$ was reported by the Bolora clinic, possibly a reflection of the high cost of making multiple trips to this rural clinic. On the other hand. Bolora had the highest follow-up visit rate $(65 \%)$ compared with an average of $48 \%$ for all 6 coalition clinics. This could reflect the lack of alternative sources of care and/or acceptability of the coalition approach to a rural population with considerable reproductive health needs.

\section{Costs}

Average total annual costs per clinic are slightly more than 51,500 taka (approx. \$2000) or 71.3 taka/MR client. If we consider direct program expenditures only the figure drops to 43,354 taka or 60 taka/MR client. There is over a 6 -fold difference in total costs between urban and rural clinics with urban clinics at the high end. A substantial part of this cost difference is related to differences in client volume. However rural clinics deliver MR services at a lower cost per client (68.3 taka vs 71.4 taka for urban clients, i.e. $\$ 2.63$ vs $\$ 2.74$ ). The urban clinics average nearly $30 \%$ of expenditures for personnel costs compared with $11 \%$ for their rural counterparts. Both rural clinics are newer and more likely to have less experienced staff with lower salaries. On the other hand, rent comprises over twice the fraction of personnel costs for rural clinics reflecting the relative scarcity of appropriate facilities in these communities. The costs of client travel time represent over $25 \%$ of total costs for rural clinics while the corresponding value for urban clinics is $15 \%$. The cost of treating medical complications comprises $1.9 \%$ of rural clinic costs compared with $2.2 \%$ for urban clinics, a reflection of the lower volume of MR services provided at rural clinics since a constant complication rate of $3 \%$ was applied in both cases. We have no evidence to suggest that the rates of clients requiring a second procedure are significantly different among all 6 clinics. The relative magnitude of cost per client ratios for urban and rural clinics contrasts with much of the cost-effectiveness literature on Third World family planning programs, which generally identifies rural programs with higher costs, a point which is discussed in a subsequent section of this paper [14].

\section{Cost-effectiveness}

The cost per MR client who leaves a coalition clinic, with a contraceptive method she has selected, is approx. 97 taka (\$3.75). Rural clinics are more cost-effective using this measure than urban clinics [73.3 taka (\$2.82) vs 99.8 taka $(\$ 3.83)$ ] a difference of $\$ 1$ per client. The cost per MR client who returns for a follow-up visit follows the same pattern [(145.1 taka (\$5.58) for rural clinics vs 147.7 taka $(\$ 5.68)$ for urban clinics] with 147.7 taka (\$5.67) as an average for all 6 clinics. The smaller cost difference reflects the relatively larger impact of travel costs for rural clients. Tabic 1 also shows cost/outcomes ratios for direct program expenditures only as a function of outcome. The differences between urban and rural clinics are further increased if this measure is used.

\section{Sensitivity analysis}

To assess the cost impact of uncertainty in the

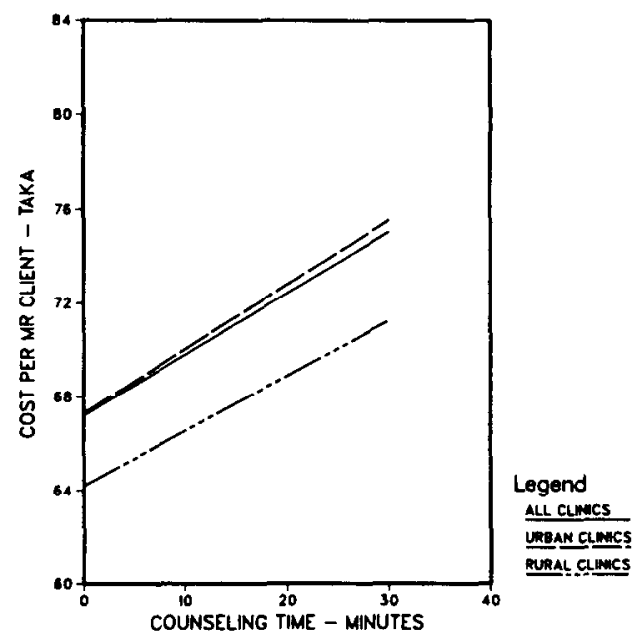

Fig. 1. The effect of counseling on cost per MR client.

estimates of personnel time attributed to counseling, total costs were calculated over a range of counseling times. These data are depicted in Fig. 1. At zero time allocated to counseling, average total costs per MR client decrease to 67.2 taka (\$2.58). If the results from Mirpur clinic are used to provide an estimate of the effect of no counseling on the percentage of clients who return for follow-up, the average cost per MR client who returns to the clinic doubles [147.7 taka $(\$ 5.68)$ to 296.2 taka (\$11.39)]. Over a range of 0 to $30 \mathrm{~min}$ average total costs per MR client increase at a rate of one taka for each 3.86 additional minutes of counseling or approx. $1 \mathrm{cent} / \mathrm{min}$. The rate is slightly higher for urban than rural clinics ( $1 \mathrm{taka} / 3.66 \mathrm{~min}$ vs 1 taka/4.27 min).

A certain amount of counseling, especially information sharing about family planning methods, also occurs during the client's time spent with the paramedic who pcrforms the MR procedure. To estimate the impact of uncertainty in time estimates for both the counselor and the paramedic, total costs were recalculated using the highest estimates for both staff members (20 min/counselor, $60 \mathrm{~min} /$ paramedic) as well as the lowest (10 min each). Average clinic total costs per MR client ranged from 77.0 taka $(\$ 2.96)$ to 63.9 taka (\$2.46) using these estimates.

\section{DISCUSSION}

Although it is extremely difficult to make costeffectiveness comparisons with other programs due to differences in general objectives and effectiveness measures, the ratios reported here are within the ranges reported for family planning programs in other developing countries which do not include menstrual regulation. To our knowledge there are no comparable economic data on other MR programs in Bangladesh or elsewhere. A study of a community based distribution program in Brazil reported a range of $\$ 3.15-\$ 5.44$ per new family planning client (roughly comparable to the MR client who leaves with a contraceptive) and \$5.84-\$9.01 per active family planning client (corresponding to the MR client returning to the clinic) depending on the population density and transportation costs [15]. This 
Table 2. A comparison of annual costs and adjusted births prevented: government of Bangladesh family planning programs and the BWHC*

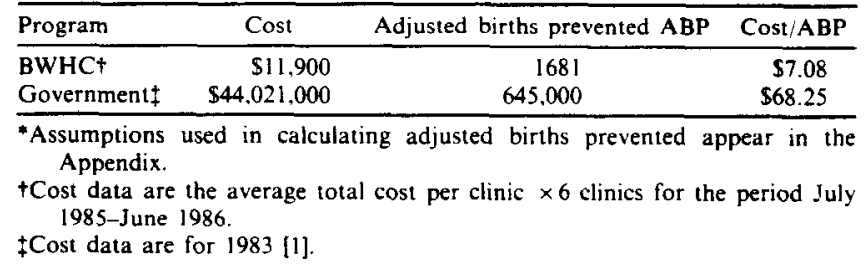

same study showed that distribution centers, which were located in hospitals and health centers, were more cost-effective than centers located in community centers and schools because they attracted more clients.

Conventional wisdom among population planners indicates that a contraceptive distribution program is more effective than a clinic-based program in rural communities because of the difficulties involved in client travel, both in terms of time and motivation. A number of field studies suggest integrated community-based distribution programs providing health, as well as family planning services, are less cost-effective than systems involving only family planning services [14]. Our data present contrasts for both hypotheses. The rural coalition clinics are more cost-effective than the urban clinics. Their costs per contracepting and continuing clients are lower than those of the urban clinics. While the rural clinics treat fewer MR clients, they are less established than their urban counterparts and their caseloads are increasing. General utilization of the rural facilities has increased at least $15 \%$ each year and the provision of an integrated program of services appears both to provide motivation for client travel to the clinics and to allow fixed costs to be spread among the various services.

The coalition's decision to expand services from MR to family planning/gynecological care, ante-natal and child health came essentially from the requests of clients and the perceptions of staff who, as women, were sensitive to the many unmet health needs faced by the clients they worked with. An understanding of the cultural reluctance in rural areas to seek personal care by women before the needs of their children are met was also an important factor in the decision to provide $\mathrm{MCH}$ care from the start in the newer, rural clinics.

A study reported by Simmons et al. using the Bangladesh government's family planning service statistics system provides a national source of comparison [16]. This study estimated the cost-effectiveness of government and private family planning programs using data describing the type and number of contraceptives distributed to cstimate the number of births averted due to contraceptive use. The authors report a cost-effectiveness ratio for all family planning programs, except USAID NGOs and social marketing programs equal to $\$ 68.25$ per adjusted birth prevented (ABP) in 1983. Comparable figures for USAID NGOs and social marketing programs were $\$ 32.20 / \mathrm{ABP}$ and $\$ 58.10 / \mathrm{ABP}$ respectively. Using the methodology of this study, the comparable figure for the coalition's program is \$7.08/ABP.
Comparative data are presented in Table 2. Assumptions used in the calculations and calculations for coalition clinics can be found in the appendix. Cost per adjusted birth prevented are calculated using the methodology employed in the Simmons et al. study The nearly 10 -fold difference in ratios must be interpreted with some caution. There are clearly differences in scale and the cost/ABP ratio for government programs is based on nationally tabulated counts of contraceptives distributed through both clinics and fieldworkers. Neither ratio includes the impact of MR on births prevented because no reliable cost data presently exist for government provided MR services. Government family planning programs do not include a counseling component. Scale factors and the provision of counseling would tend to increase the cost ratio of $B W H C$ relative to government programs while the relative impact of $M R$ as a birth prevention method cannot be estimated. However, it seems safe to say that the coalition program is at least as cost-effective as any existing family planning program in the country at present

Government expenditures for family planning in the 1986-87 fiscal year are reported to be 1.2 billion taka (\$46.5 million) or approx. 36 taka/woman per year in the age group 15-44 years. Obviously many women do not have access to family planning services so the actual expenditure per woman who receives services is much greater. From a national policy perspective on MR services, the relative cost-effectiveness of the coalition's program with its emphasis on counseling and the Bangladesh government's delivery of MR needs to be determined but is beyond the scope of the study reported here. One practical obstacle is the difficulty in describing client use of MR and other reproductive health services over a period of time because most MR providers, private and government sponsored, organize record keeping based on the types of services delivered BWHC has recently reorganized its record-keeping system to a client-centered one making possible periodic monitoring of the continuity of care received over time and providing answers to questions about how rural clients use clinic-based reproductive health care services. This will be especially useful for assessing longer term aspects of post-MR client behavior regarding family planning and reproductive health care in general.

Acknowledgements-The authors would like to thank Adrienne Germain, Judith Bruce and Ruth Dixon-Mueller for their helpful comments on an earlier draft of this paper. This study was supported by the International Women's Health Coalition, New York. 


\section{REFERENCES}

1. Akher $\mathrm{H}$. Abortion in Bangladesh. Bangladesh $J$. Obstet. Gynaec. 1-14, April 1986.

2. Rochat R., Jabeen S. and Rosenberg M. Maternal and abortion-related deaths in Bangladesh 1978-79. Int. $J$. Gynaec. Obstet. 19, 155-164. 1981.

3. Measham A., Obaidullah M. and Rosenberg M. Complications from induced ahortion in Bangladesh related to types of practitioners and methods and impact on mortality. The Lancet 199-202, 24 January, 1981.

4. Bhuiyan S. and Burkhart M. Maternal and public health benefits of menstrual regulation in Chittagong. Int. J. Gynaec. Obstet. 20, 105-109, 1982.

5. Begum S., Kamal H. and Kamal G. Evaluation of $M R$ Services in Bangladesh, p. 2. Bangladesh Association for the Prevention of Septic Abortion, Dhaka, 20 May, 1987.

6. Kay B. J. Evaluating the quality of ambuiatory abortion services: a cost-effectiveness approach. In Systems Science in Health Care (Edited by Tilquin C.), pp. 351-358. Pergamon Press, Toronto, 1981.

7. Begum S., Kamal H., Khanam S. and Kamal G. Evaluation of $M R$ Services and Training Programs, pp. 2 3. Bangladesh Association for Prevention of Septic Abortion, Dhaka, 1985

8. Anonymous. Clinic staff's insensitive attitudes deter low-caste Nepalese women from practicing family planning. Fam. Planng Perspect. 12(1), 31, 33, March 1986.

9. Statement by a Coalition of Third World Women and
Friends of Third World Women; read at the United Nation's World Conference on Women. Nairobi. Kenya, 18 July, 1985.

10. Kay B. and Thompson C. An outcome evaluation of counseling services provided by abortion clinics. Med. Care 15, 858-868, 1977.

11. Braken M., Grossman G., Hachamovitch H., Sussman $D$. and Schrieir D. Abortion counseling: an experimental study of three techniques. J. Obstet. Gynec. 117 . 10-20, September 1973.

12. Personal communication. Deborah Balk. Dept of Population Planning and International Health, School of Public Health, University of Michigan, October 1986.

13. Chowdhury G. Baseline Survev of the Bolora, Palash and Mirpur Project Areas. BWHC, Dhaka, 1985.

14. Population Reports. Cost-effectiveness of programs, Series J, Number 31, Vol. XIV, No. 2, J 834-835, May-June 1986.

15. Foreit J. R., Rodriques W., Arruda J. M. and Milare J A cost effectiveness comparison of service delivery systems and geographic areas in Paqui State, Brazil. In Evaluating Population Programs (Edited by Sirageldin I., Salkever D. and Osborn R.), pp. 406-420. Croom Helm, London, 1983.

16. Simmons G., Rob U. and Bernstein S. An economic analysis of family planning in Bangladesh, A RAPID Project Report, Dept of Population Planning and International Health, The University of Michigan, Tables 2. 13. February 1986.

\section{APPENDIX}

Table assumptions used for calculating adjusted births prevented*:

\begin{tabular}{lrrccc}
\hline Method & $\begin{array}{c}\text { Units per } \\
\text { CYPt }\end{array}$ & $\begin{array}{c}\text { CYPs per } \\
\text { BP }\end{array}$ & $\begin{array}{c}\text { Units per } \\
\text { BP }\end{array}$ & $\begin{array}{c}\text { Discount } \\
\text { rate }\end{array}$ & $\begin{array}{c}\text { Adjustment } \\
\text { factor }\end{array}$ \\
\hline Oral pill & 14.00 & 3.5 & 49.000 & 15 & 0.87 \\
Condom & 300.00 & 3.5 & 1050.000 & 15 & 0.87 \\
Injection & 4.00 & 3.5 & 14.000 & 15 & 0.87 \\
IUD/Cu T & 2.45 & 3.5 & 0.70 & 15 & 0.756 \\
Tubectomy & 7.75 & 3.5 & 2.21 & 15 & 0.572 \\
\hline
\end{tabular}

*Adapted from Simmons G. B. et al. Summary Views on the Economics of Family Planning in Bangladesh, A RAPID Project Report, Table 2, p. 18. Table 2, February 1986.

$+\mathrm{CYP}=$ couple-years of protection

¥Average expected lifetime of effects of method used in calculating adjustment factors: pill, condom and injection $=1.0$ year; IUD/copper $\mathrm{T}=2.0$ years; tubectomy $=4.0$ years

Adjusted births prevented calculations for the BWHC

\begin{tabular}{lcccc}
\hline Method & $\begin{array}{c}\text { Total units } \\
\text { dispensed } \\
(\mathrm{A})\end{array}$ & $\begin{array}{c}\text { Units/birth } \\
\text { prevented } \\
(\mathrm{B})\end{array}$ & $\begin{array}{c}\text { Adjustment } \\
\text { factor } \\
(\mathrm{C})\end{array}$ & $\begin{array}{c}\text { Adjusted births } \\
\text { prevented } \\
\text { (A/B)C }\end{array}$ \\
\hline Oral pill & 3558 & 49.00 & 0.87 & 63.173 \\
Condom & 14.175 & 1050.00 & 0.87 & 11.745 \\
Injection & 250 & 14.00 & 0.87 & 15.536 \\
IUD/Cu T & 1445 & 0.70 & 7.56 & 1560.6 \\
Tubectomy & 115 & 2.21 & 0.572 & 29.765 \\
Total adjusted births prevented & & 1680.819 \\
\hline
\end{tabular}

